\title{
Significance of Pattern of Invasion in Tongue Squamous Cell Carcinoma-A Retrospective Study from a Regional Cancer Center of North-East India
}

\author{
Yopovinu Rhutso $^{1}$ Lopa M. Kakoti ${ }^{1}$ Jagannath D. Sharma ${ }^{1}$ Manoj Kalita ${ }^{2}$
}

1 Department of Oncoathology, Dr. B.Borooah Cancer Institute, Guwahati, Assam, India; ${ }^{2}$ Department of Epidemiology and Statistics, Dr. B.Borooah Cancer Institute, Guwahati, Assam, India

South Asian J Cancer 2022;11(2):140-145.
Address for correspondence Lopa M. Kakoti, MD, DNB, Department of Oncopathology, Dr. B. Borooah Cancer Institute, Gopinath Nagar, Guwahati, Assam-781016, India

(e-mail: lopamudrakakoti@gmail.com).

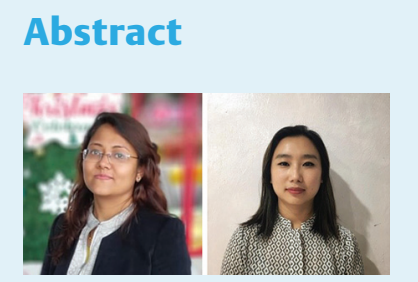

Introduction Many multiparametric models and scoring systems had been proposed in oral tongue squamous cell carcinoma (OTSCC) to predict the survival and recurrence, out of which pattern of invasion (POI) is gaining focus recently.

Objective We conducted a retrospective study with the aim to see the prognostic significance of the $\mathrm{POI}$ and other parameters such as depth of invasion, perineural invasion (PNI), and lymph node status in OTSCC.

Materials and Methods The slides of already diagnosed OTSCC cases were prepared from January 2015 to December 2017 records and studied by two pathologists for different patterns using Brandwein-Gensler scoring system. The different clinicopathologic parameters were compared with different POI. Statistical analysis was performed to present cumulative survival outcomes and for comparison.

Results Most of our patients were $>40$ years of age with male preponderance. Tumor differentiation pattern was assessed using Broders' system of grading that showed $85 \%$ of tumors were well, $12.5 \%$ tumors were moderately differentiated, and $2.5 \%$ tumors were poorly differentiated. The worst POI was predominantly pattern III (50\%) followed by pattern II (32.5\%) and pattern IV (13.8\%). Most patients were in stage II (33.8\%)

Keywords followed by stage III (32.5\%), stage IV (26.3\%), and stage I (7.5\%). There was an inverse relationship between $\mathrm{POI}$ with tumor stage and recurrence. A strong statistically significant association was found between $\mathrm{POI}$ with perineural infiltration and lymph node metastasis.

Conclusion We concluded from our study that worst POI is significantly associated with number of lymph nodes metastasis and perineural infiltration and hence can be used as an independent prognostic factor.
DOI https://doi.org/10.1055/s-0041-1736201 ISSN 2278-330X

How to cite this article: Rhutso Y, Kakoti LM, Sharma JD, et al. Significance of Pattern of Invasion in Tongue Squamous Cell Carcinoma-A Retrospective Study from a Regional Cancer Center of North-East India South Asian J Cancer 2022;11(2):

$140-145$.
(C) 2022. MedIntel Services Pvt Ltd. All rights reserved.

This is an open access article published by Thieme under the terms of the Creative Commons Attribution-NonDerivative-NonCommercial-License, permitting copying and reproduction so long as the original work is given appropriate credit. Contents may not be used for commercial purposes, or adapted, remixed, transformed or built upon. (https://creativecommons.org/licenses/by-nc-nd/ 4.0/)

Thieme Medical and Scientific Publishers Pvt. Ltd., A-12, 2nd Floor, Sector 2, Noida-201301 UP, India 


\section{Introduction}

Broders first introduced in 1920 histopathological grading of squamous cell carcinoma (SCC) based on the proportion of differentiated cells to undifferentiated or anaplastic cells within the entire tumor cell population and graded as highly/well, moderately, poorly differentiated, and undifferentiated tumors. ${ }^{1,2}$ The utility of tumor differentiation using Broders' histopathological malignancy grading system in predicting the clinical course and outcome of oral tongue squamous cell carcinoma (OTSCC) has been found to be limited and criticized because of its poor prognostic value, and lack of reproducibility, underscoring the need for more complex grading systems. Studies by Brandwein-Gensler et $\mathrm{al}^{3}$ Almangush $\mathrm{A}$ et $\mathrm{al}^{4}{ }^{4}$ Anneroth et $\mathrm{al}^{5}{ }^{5}$ Bryne et $\mathrm{al},{ }^{6}$ and Martínez-Gimeno et $\mathrm{al}^{7}$ came into being where multiparameter prognostic models and scoring systems that include nuclear pleomorphism, mitotic index, lymphocytic response, tumor growth pattern, tumor thickness, degree of keratinization, depth of invasion, and pattern of invasion (POI) have been proposed ${ }^{7-10}$ to predict the survival of patients with OTSCC and there was a general agreement that the most useful prognostic information can be deduced from the invasive front of the tumor, where the deepest and presumably most aggressive cells reside. ${ }^{11}$

SCC was encountered as the most common histological diagnosis in the malignant neoplasm of tongue; we conducted a retrospective study on 80 patients with an aim to study the prognostic significance of the POI and other clinicopathological parameters such as survival, stage, recurrence, depth of invasion, perineural invasion, and lymph node status in OTSCC.

\section{Materials and Methods}

\section{Tissue Specimens}

Paraffin-embedded sections of 80 patients with OTSCC collected between January 2015 and December2017 from the hospital archives were evaluated. Only exclusion criteria used were any patients receiving preoperative chemo- or radiotherapy and recurrent cases. All the sections were from the surgical resection specimen. Patients with history of any prior treatment for OTSCC, residual or recurrent cases, and biopsy specimens were excluded.

\section{Histological Evaluation}

All the hematoxylin and eosin-stained histopathological slides were concurrently reviewed and evaluated independently by two qualified pathologists (the first observer LMK and the second observer YR) using the same type of microscope without any prior knowledge of each patient's clinical details. All the hematoxylin and eosin-stained histopathological slides were concurrently reviewed and evaluated independently by two qualified pathologists (the first observer and the second observer) using the same type of microscope without any prior knowledge of each patient's clinical details. A set criterion was formulated for the evaluation of the slides as follows: a. All the sections of the tumor were evaluated microscopically by the two pathologists (LMK and YR).

b. The maximum depth of infiltration was calculated microscopically under $40 \mathrm{x}$ and would be taken for consideration

c. The POI was taken for consideration in the section of maximum depth of thickness at its infiltrating edge following the criteria set by Brandwein-Gensler et $\mathrm{al}^{3}$ (details mentioned in the next paragraph) under high power resolution (40X).

d. Extratumoral angiolymphatic invasion as mentioned in College of American Pathologist (CAP) guidelines was not taken into consideration as worst POI (WPOI) 5 in this study.

When the opinions of the two evaluators differed selecting the sections or maximum depth of invasion, consensus was reached by discussion.

\section{Histologic Variables and Invasion Pattern Grading}

Tumor differentiation was done using Broders' grading system. Variables like depth of invasion, lymph node metastasis, perineural invasion, and POI were evaluated.

Depth of invasion was measured from the base of adjacent normal epithelium to the deepest point of invasive tumor.

Tumor POI was examined at the host-tumor interface. Invasive tumor front scoring system defined by BrandweinGensler et $\mathrm{al}^{3}$ was used in our study comprising patterns I to $\mathrm{V}$. Pattern I represents tumor invasion in a broad pushing manner. Pattern II represents tumor invasion with broad pushing "fingers," or separate large tumor islands, with a stellate appearance. Pattern III represents invasive islands of tumor $>15$ cells per island. Pattern IV represents invasive tumor islands $<15$ cells per island, including single cell invasion and single-cell filing pattern. Pattern $\mathrm{V}$ represents tumor satellites of any size at distance of $1 \mathrm{~mm}$ or more from intervening normal tissue (not fibrosis) at the invasive tumor front.

\section{Statistical Analysis}

Frequency tables of demographic and clinicopathological parameters were established. Kaplan-Meier plot was constructed to present cumulative survival outcomes and compared using the log-rank test. A $p$-value of $<0.05$ was considered to be statistically significant.

\section{Results}

The distribution of patients by demographic and clinicopathologic factors is shown in -Table 1. The mean followup period was 35 months (range: 1-60 months). Out of the 80 patients, 18 patients died of OTSCC because of recurrence, whereas 40 were alive at the end of the follow-up period, while remaining 22 patients were lost to follow-up.

OTSCC was more common in patients age $\geq 40$ years (82.5\%) in comparison to $<40$ years $(17.5 \%)$. It had male preponderance $(71.3 \%)$ over female $(28.7)$ with male to female ratio of $2.5: 1$. 
Table 1 Demographic and clinicopathological features of 80 patients with oral tongue squamous cell carcinoma

\begin{tabular}{|l|l|l|l|l|l|}
\hline Parameters & Number of patients & $\mathbf{( \% )}$ & Parameters & Number of patients & (\%) \\
\hline Age & & & Perineural invasion & & \\
\hline$<40$ years & 14 & 17.5 & Present & 31 & 38.7 \\
\hline$\geq 40$ years & 66 & 82.5 & Absent & 49 & 61.3 \\
\hline Gender & & & Lymph Node Metastasis & & \\
\hline Male & 57 & 71.3 & Present & 31 & 38.7 \\
\hline Female & 23 & 28.3 & Absent & 49 & 61.3 \\
\hline M:F & $2.5: 1$ & & & & \\
\hline Differentiations & & & Recurrence & \\
\hline WDSCC & 68 & 85 & Present & 18 & 22.5 \\
\hline MDSCC & 10 & 12.5 & Absent & 62 & 77.5 \\
\hline PDSCC & 2 & 2.5 & & & \\
\hline Status & & & Tumor Depth & \\
\hline Alive & 40 & 50 & $\leq 5 \mathrm{~mm}$ & 22 & 27.5 \\
\hline Dead & 18 & 22.5 & $>5-10 \mathrm{~mm}$ & 39 & 48.8 \\
\hline Lost to follow-up & 22 & 27.5 & $>10 \mathrm{~mm}$ & 19 & 23.8 \\
\hline Stage & & & Pattern & & \\
\hline Stage I & 6 & 7.5 & Pattern I & 0 & 13.8 \\
\hline Stage II & 27 & 33.8 & Pattern II & 11 & 50.0 \\
\hline Stage III & 26 & 32.5 & Pattern III & 40 & 32.5 \\
\hline Stage IV & 21 & 26.3 & Pattern IV & 26 & 3.8 \\
\hline & & & Pattern V & 3 & \\
\hline
\end{tabular}

Abbreviations: MDSCC, moderately differentiated squamous cell carcinoma; PDSCC, poorly differentiated squamous cell carcinoma; WDSCC, worst differentiated squamous cell carcinoma.

Tumor differentiation pattern assessed using Broders' system of grading showed that $85 \%$ of tumors were well differentiated, $12.5 \%$ tumors were moderately differentiated, and $2.5 \%$ tumors were poorly differentiated.

Tumor depth of $>5$ to $10 \mathrm{~mm}$ was seen in $48.8 \%$ followed by $\leq 5 \mathrm{~mm}$ in $27.5 \%$ and $>10 \mathrm{~mm}$ in $23.8 \%$ of patients.

The different patterns were predominantly pattern III (50\%) followed by pattern II (32.5\%), pattern IV (13.8\%), and the least was pattern $\mathrm{V}(3.8 \%)$. The pattern I was not seen in our study (-Fig. 1).

Most patients were in stage II (33.8\%) followed by stage III (32.5\%) and stage IV (26.3\%). Least common was stage I (7.5\%).

Perineural invasion and lymph node metastasis were seen in $38.7 \%$ each, while recurrence occurred in $22.5 \%$ of patients.

\section{Survival Outcomes}

The overall survival of patients with OTSCC at 1 year, 3 years, and 5 years was $92.9,58.5$, and 50.5 , respectively. Depth of invasion, perineural invasion, nodal metastasis, and POI correlate well with the overall survival in patients with OTSCC; however, no statistically significance $(p<0.05)$ was seen.

There was an inverse relationship between POI with tumor stage and recurrence; however, it did not show strong statistical association (-Table 2).

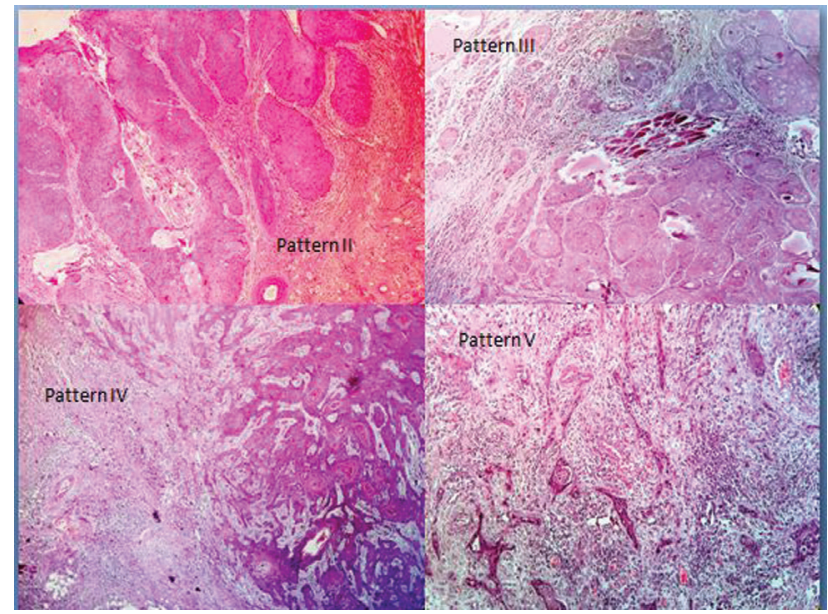

Fig. 1 The different patterns of infiltration, hematoxylin and eosin, 20X.

A strong statistically significant association was found between POI with perineural infiltration $(<0.013)$ and lymph node metastasis $(<0.0001 ;-$ Table 3$)$.

\section{Discussion}

The present study assessed the effectiveness of clinicopathological parameters in predicting the mortality as well as the 


\section{POI vS STAGE}

\begin{tabular}{|c|c|c|c|c|c|c|c|}
\hline & & & Stage & & & & \\
\hline & & stagel & Stagell & Staze III & stage iv & iotad & \\
\hline \multirow[t]{8}{*}{ pattern } & II & 3. & 6 & 2 & 0 & 11 & \\
\hline & & $50,0 \%$ & $20.20 \%$ & $7.70 \%$ & $0,0 \%$ & $13.20 \%$ & \\
\hline & II & 기 & 15 & 14 & 8 & 40 & \\
\hline & & $\operatorname{son} \%$ & $55.60 \%$ & $53.80 \%$ & $2810 \%$ & $50.00 \%$ & \\
\hline & N & d. & 6 & 9 & 11 & 26 & 0.17 \\
\hline & & $0,0 \%$ & $22.20 \%$ & $34.66 \%$ & $32.40 \%$ & $32.50 \%$ & \\
\hline & y & 0 & 0 & 1 & 2 & 3 & \\
\hline & & $0,00 \%$ & $0.00 \%$ & $3.80 \%$ & $9.50 \%$ & $3.80 \%$ & \\
\hline \multirow[t]{2}{*}{ Total } & & 6 & 27 & 26 & 21 & 8) & \\
\hline & & $10000 \%$ & $100.00 \%$ & $100.00 \%$ & $100.00 \%$ & $100.00 \%$ & \\
\hline
\end{tabular}

\section{POI VS Recurrence}

\begin{tabular}{|c|c|c|c|c|c|}
\hline & & \multicolumn{2}{|l|}{ Recurrence } & \multirow[b]{2}{*}{ Total } & \\
\hline & & No & Yes & & \\
\hline \multirow[t]{8}{*}{ pattern } & II & 11 & 0 & 11 & \\
\hline & & $17.70 \%$ & $0.00 \%$ & $13.80 \%$ & \\
\hline & III & 28 & 12 & 40 & 0.147 \\
\hline & & $45.20 \%$ & $66.70 \%$ & $50.00 \%$ & \\
\hline & IV & 20 & 6 & 26 & \\
\hline & & $32.30 \%$ & $33.30 \%$ & $32.50 \%$ & \\
\hline & v & 3 & 0 & 3 & \\
\hline & & $4.80 \%$ & $0.00 \%$ & $3.80 \%$ & \\
\hline Total & & 62 & 18 & 81 & \\
\hline & & $100.00 \%$ & $100.00 \%$ & $100.00 \%$ & \\
\hline
\end{tabular}

Table 2 Relationship between pattern of invasion (POI) on oral tongue squamous cell carcinoma with stage and recurrence.

\section{POI vs PNI}

\begin{tabular}{|l|l|r|r|r|r||}
\hline \multicolumn{7}{|c|}{ PNI } & & \\
\hline & & absent & present & Total & p value \\
\hline pattem & II & 9 & 2 & 11 & \\
\hline & & $18.40 \%$ & $6.50 \%$ & $13.80 \%$ & \\
\hline & III & 29 & 11 & 40 & \\
\hline & & $59.20 \%$ & $35.50 \%$ & $50.00 \%$ & \\
\hline & IV & 10 & 16 & 26 & 0.013 \\
\hline & & $20.40 \%$ & $51.60 \%$ & $32.50 \%$ & \\
\hline & V & 1 & 2 & 3 & \\
\hline & & $2.00 \%$ & $6.50 \%$ & $3.80 \%$ & \\
\hline Total & & 49 & 31 & 80 & \\
\hline & & $100.00 \%$ & $100.00 \%$ & $100.00 \%$ & \\
\hline
\end{tabular}

POI vs LN status

\begin{tabular}{|l|l|l|l|l|l|}
\hline & & LN & & & \\
\hline & & Absent & Present & Total & \\
\hline pattern & II & 11 & 0 & 11 & \\
\hline & & $22.40 \%$ & $0.00 \%$ & $13.80 \%$ & \\
\hline & III & 28 & 12 & 40 & \\
\hline & & $57.10 \%$ & $38.70 \%$ & $50.00 \%$ & \\
\hline & IV & 10 & 16 & 26 & $<0.0001$ \\
\hline & & $20.40 \%$ & $51.60 \%$ & $32.50 \%$ & \\
\hline & V & 0 & 3 & 3 & \\
\hline & & $0.00 \%$ & $9.70 \%$ & $3.80 \%$ & \\
\hline Total & & 49 & 31 & 80 & \\
\hline & & $100.00 \%$ & $100.00 \%$ & $100.00 \%$ & \\
\hline \hline
\end{tabular}

Table 3 Relationship between pattern of invasion (POI) on oral tongue squamous cell carcinoma with perineural invasion (PNI) and lymph node (LN) metastasis.

prognostic significance of the POI at the host-tumor interface of patients with OTSCC.

In the present study, the overall survival of patients with OTSCC at 1 year, 3 years, and 5 years was 92.9, 58.5, and 50.5, respectively. A reduced 5-year survival has been reported by Spiro et al for patients with oral tongue cancer. ${ }^{12,13}$ We did not find a statistically significant relationship among the depth of invasion, lymph node metastasis, and perineural invasion with patient mortality. The depth of invasion by microscopic method is best evaluated only after the patient has undergone surgical treatment of the tumor and hence the usefulness in prognostication is limited. Studies done in early stage OTSCC found depth of invasion to be an independent prognostic factor. A preoperative determination of tumor depth through MRI and ultrasonography has been suggested by several groups. ${ }^{14,15}$ Possibly the small sample size and inclusion of both early (T1-T2) and late stage(T3-T4) OTSCC in the present study would explain the reason why a statistical significance was not found between depth of invasion, lymph node metastasis, and perineural invasion with overall survival.

Majority of the cases in the present study were welldifferentiated SCC (85\%) and no association of differentiation with the POI was found. This was also observed in study done by Nadaf et al, ${ }^{16}$ and concluded that five POIs could serve as an individual prognostic marker irrespective of the histologic differentiation of tumor. Different POIs have been reported for SCCs of different sites, for example, the skin, head and neck, as well as the cervix uteri SCCs and gastric and endometrial adenocarcinomas, ${ }^{17-20}$ and a high grade of 
tumor cell dissociation at the invasive front has been reported to be of prognostic value in different types of carcinomas. Several studies in OTSCC have previously confirmed the relationship of unfavorable WPOI with poor prognosis in OTSCC. ${ }^{11,21}$

Bryne et al reported grading system for invasive front that was found to have prognostic significance. ${ }^{9}$ In a study by Heerema et al showed that the POI (one of the features of Bryne's malignancy grading system) is an independent prognostic factor in low-stage OSCC. ${ }^{22}$ Nadaf et al showed that five POIs (one component of invasive tumor front) could serve as an individual prognostic marker irrespective of the histologic differentiation of tumor. ${ }^{16}$ In a study by Almangush et al, tumor budding, the depth of invasion (DOI), and WPOI were evaluated and it was found that they are significant prognostic markers for early stage (T1 and T2, N0 M0) carcinomas of tongue. ${ }^{23}$

In our study, it was observed that recurrence was most commonly seen in pattern III followed by pattern IV and there was no recurrence in pattern II. Many cases from pattern $\mathrm{V}$ were lost to follow-up. However, there was an inverse relationship between POI with tumor stage and recurrence, but it did not show strong statistical association, probably due to smaller sample size as well as many patients with highest POI were lost to follow-up. Similarly, Monteiro et al reported that the pattern of spread in oral cancers show little or no relation to the clinical stage at presentation. ${ }^{24}$ However, in another study by Odell et al on 42 OTSCC, the prognostic significance of the invasive front grading and the close correlation between POI and metastasis as well as any recurrence of the disease were demonstrated. ${ }^{25}$

We found that LN metastasis and PNI strongly correlate with the worst POI and were found to be statistically significant. PNI was most commonly seen in association with $66.7 \%$ of pattern $\mathrm{V}$ followed by $61.5 \%$ of pattern IV and $27.5 \%$ in pattern III, and $18 \%$ of pattern II. Lymph node metastasis was seen in $100 \%$ cases of pattern $V$ followed by $61.5 \%$ of pattern IV and in $30 \%$ of pattern III, but no lymph node metastasis was seen in pattern II. Several studies also support the association between POI and lymph node metastasis. ${ }^{25,26}$ However, we have not found any studies that show association between perineural infiltration with the pattern of invasion. Hence, we feel the need of to test further in other studies to substantiate this strong association found in our study.

\section{Conclusion}

In conclusion, as observed collectively from different studies mentioned above, it is well known that the most aggressive cells of tumor reside at the invasive front of tumor and show poorer prognosis in relation to worse pattern of invasion at the invasive front of tumor. The present study concluded that POI IV and V are significantly associated with number of lymph nodes metastasis and perineural infiltration, and hence can be an independent histopathologic prognostic parameter in OTSCC.
Source of Support

Nil.

Conflict of Interest

None.

\section{Acknowledgment}

Our technicians for their support.

\section{Reference}

1 Broders AC. Squamous cell epithelioma of the lip. JAMA 1920; 74:656-4

2 Broders AC. The microscopic grading of cancer. Surg Clin North Am 1941;21:947-6

3 Brandwein-Gensler M, Teixeira MS, Lewis CM, et al. Oral squamous cell carcinoma: histologic risk assessment, but not margin status, is strongly predictive of local disease-free and overall survival. Am J Surg Pathol 2005;29(02):167-178

4 Almangush A, Bello IO, Keski-Säntti H, et al. Depth of invasion, tumor budding, and worst pattern of invasion: prognostic indicators in early-stage oral tongue cancer. Head Neck 2014;36(06): 811-818

5 Anneroth G, Batsakis J, Luna M. Review of the literature and a recommended system of malignancy grading in oral squamous cell carcinomas. Scand J Dent Res 1987;95(03):229-249

6 Bryne M, Koppang HS, Lilleng R, Stene T, Bang G, Dabelsteen E. New malignancy grading is a better prognostic indicator than Broders' grading in oral squamous cell carcinomas. J Oral Pathol Med 1989;18(08):432-437

7 Martínez-Gimeno C, Rodríguez EM, Vila CN, Varela CL. Squamous cell carcinoma of the oral cavity: a clinicopathologic scoring system for evaluating risk of cervical lymph node metastasis. Laryngoscope 1995;105(7 Pt 1):728-733

8 Hiratsuka H, Miyakawa A, Nakamori K, Kido Y, Sunakawa H, Kohama G. Multivariate analysis of occult lymph node metastasis as a prognostic indicator for patients with squamous cell carcinoma of the oral cavity. Cancer 1997;80(03):351-356

9 Bryne M, Koppang HS, Lilleng R, Kjaerheim A. Malignancy grading of the deep invasive margins of oral squamous cell carcinomas has high prognostic value. J Pathol 1992;166(04):375-381

10 Kane SV, Gupta M, Kakade AC, D' Cruz A. Depth of invasion is the most significant histological predictor of subclinical cervical lymph node metastasis in early squamous carcinomas of the oral cavity. Eur J Surg Oncol 2006;32(07):795-803

11 Chang YC, Nieh S, Chen SF, Jao SW, Lin YL, Fu E. Invasive pattern grading score designed as an independent prognostic indicator in oral squamous cell carcinoma. Histopathology 2010;57(02):295-303

12 Spiro RH, Guillamondegui O Jr, Paulino AF, Huvos AG. Pattern of invasion and margin assessment in patients with oral tongue cancer. Head Neck 1999;21(05):408-413

13 Hoşal AS, Unal OF, Ayhan A. Possible prognostic value of histopathologic parameters in patients with carcinoma of the oral tongue. Eur Arch Otorhinolaryngol 1998;255(04):216-219

14 Jung J, Cho NH, Kim J, et al. Significant invasion depth of early oral tongue cancer originated from the lateral border to predict regional metastases and prognosis. Int J Oral Maxillofac Surg 2009;38(06):653-660

15 Mark Taylor S, Drover C, Maceachern R, et al. Is preoperative ultrasonography accurate in measuring tumor thickness and predicting the incidence of cervical metastasis in oral cancer? Oral Oncol 2010;46(01):38-41

16 Nadaf A, Bavle RM, Soumya M, D'mello S, Kuriakose MA, Govindan S. Analysis of the invasive edge in primary and secondary oral squamous cell carcinoma: An independent prognostic marker: a 
retrospective study. J Oral Maxillofac Pathol 2016;20(02): 239-245

17 Gabbert HE, Meier S, Gerharz CD, Hommel G. Tumor-cell dissociation at the invasion front: a new prognostic parameter in gastric cancer patients. Int J Cancer 1992;50(02):202-207

18 Suzuki C, Matsumoto T, Sonoue H, Arakawa A, Furugen Y, Kinoshita K. Prognostic significance of the infiltrative pattern invasion in endometrioid adenocarcinoma of the endometrium. Pathol Int 2003;53(08):495-500

19 Olsen KD, Caruso M, Foote RL, et al. Primary head and neck cancer. Histopathologic predictors of recurrence after neck dissection in patients with lymph node involvement. Arch Otolaryngol Head Neck Surg 1994;120(12):1370-1374

20 Kristensen GB, Abeler VM, Risberg B, Trop C, Bryne M. Tumor size, depth of invasion, and grading of the invasive tumor front are the main prognostic factors in early squamous cell cervical carcinoma. Gynecol Oncol 1999;74(02):245-251

21 Dissanayaka WL, Pitiyage G, Kumarasiri PV, Liyanage RL, Dias KD, Tilakaratne WM. Clinical and histopathologic parameters in survival of oral squamous cell carcinoma. Oral Surg Oral Med Oral Pathol Oral Radiol 2012;113(04):518-525
22 Heerema MG, Melchers LJ, Roodenburg JL, Schuuring E, de Bock $\mathrm{GH}$, van der Vegt B. Reproducibility and prognostic value of pattern of invasion scoring in low-stage oral squamous cell carcinoma. Histopathology 2016;68(03):388-397

23 Almangush A, Bello IO, Keski-Säntti H, et al. Depth of invasion, tumor budding, and worst pattern of invasion: prognostic indicators in early-stage oral tongue cancer. Head Neck 2014;36(06): 811-818

24 Monteiro LS, Amaral JB, Vizcaíno JR, Lopes CA, Torres FO. A clinical-pathological and survival study of oral squamous cell carcinomas from a population of the North of Portugal. Med Oral Patol Oral Cir Bucal 2014;19(02):e120-e126

25 Odell EW, Jani P, Sherriff M, et al. The prognostic value of individual histologic grading parameters in small lingual squamous cell carcinomas. The importance of the pattern of invasion. Cancer 1994;74(03):789-794

26 Akhter M, Hossain S, Rahman QB, Molla MR. A study on histological grading of oral squamous cell carcinoma and its co-relationship with regional metastasis. J Oral Maxillofac Pathol 2011;15 (02):168-176 